\title{
LESSONS LEARNED FROM NASA UAV SCIENCE DEMONSTRATION PROGRAM MISSIONS
}

\author{
Steven S. Wegener, Program Manager \\ NASA Ames Research Center, Moffett Field, California \\ Susan M. Schoenung, Project Engineer \\ Longitude 122 West, Inc., Menlo Park, California
}

\begin{abstract}
During the summer of 2002, two airborne missions were flown as part of a NASA Earth Science Enterprise program to demonstrate the use of uninhabited aerial vehicles (UAVs) to perform earth science. One mission, the Altus Cumulus Electrification Study (ACES), successfully measured lightning storms in the vicinity of Key West, Florida, during storm season using a highaltitude Altus ${ }^{T M}$ UAV. In the other, a solar-powered UAV, the Pathfinder Plus, flew a high-resolution imaging mission over coffee fields in Kauai, Hawaii, to help guide the harvest.
\end{abstract}

In this paper, lessons learned with respect to the use of UAVs in science missions are presented in these areas:

- Performance of two state-of-the-art UAVs

- Payload requirements for remotely controlled data collection

- Operation in National Air Space

- Mission management

Considerations for future technology development and NASA UAV missions are also discussed.

\section{Background}

NASA Research Announcement NRA 00-OES-02 (UAV Science Demonstration Program) was issued in 2000 . The objectives of the research program were to:

- Conduct high quality research that exploited the unique capabilities of UAV aircraft.

- Demonstrate the utility and promise of UAV platforms for Earth Science observations and measurements.

- Build confidence in UAV platforms through scientifically useful demonstrations.

Two missions were selected based on the criteria for the NRA, including science value, UAV-unique contribution, risk, and cost. Grants were awarded in the spring of 2001. Following a pre-deployment phase, both field campaigns took place in mid2002. This paper highlights lessons learned from the pre-deployment and deployment phases. Post-deployment data analysis activities are still ongoing.

The Altus Cumulus Electrification Study (ACES) was led by a team from NASA Marshall Space Flight Center (MSFC). They flew the General Atomics Altus ${ }^{\mathrm{TM}} \cup A V$ in pursuit of electric field and other measurements in the vicinity of lightning storms. Their base of operations was Naval Air Facility Key West (NAFKW).

The UAV Coffee Harvest Optimization experiment was led by Clark University and team members from NASA Ames Research Center (ARC). They flew the solar-powered AeroVironment Pathfinder Plus over coffee fields in Kauai to determine from visible and infrared signatures the ripeness of coffee cherries in individual fields. This information was used, in turn, to optimize the harvest schedule. The Pathfinder Plus flew from Pacific Missile Range Facility (PMRF) on the island of Kauai.

Lessons Learned documentation was prepared by the science teams, with input from their UAV suppliers. The objectives of reporting Lessons Learned are to:

- Provide guidance to future users on the use of UAVs for science and applications.

- Identify needs and provide recommendations for future research and development of platforms, sensors, and systems.

- Provide feedback to UAV builders and service providers for future missions.

\section{Science Accomplishments}

Both missions were highly successful in achieving their science and application goals. In August 2002 the ACES experiment flew 13 flights, collecting over thirty hours of electric field and 
meteorological data in the vicinity of thunderstorms in south Florida. The Altus ${ }^{\mathrm{TM}}$ UAV was able to fly over and around the weather of interest because of its altitude and endurance capabilities. It is also electrically quiet, a good feature for meteorological missions. A unique feature of the ACES mission was the ability to indicate the location of the aircraft on a display of real-time weather. The software for this visual aid, developed at MSFC, is now available for other users, UAVs or other aircraft. A view of the display screen is shown in Figure 1. More details about the science experiment can be found in References 1 and 2.

The UAV Coffee mission was designed to demonstrate the value of a long endurance, slow speed platform in remote sensing applications. The September 2002 experiment targeted agriculture specifically. Because of the loitering ability of the Pathfinder Plus, the UAV Coffee mission was able to collect cloud-free images of the Kauai Coffee plantation, even though it was a day with $70 \%$ cloud cover, by manipulating the aircraft to openings in the clouds over a period of several hours. Ripeness indicators were derived from the images in near-real time. The plantation owner made direct use of the ripeness data and information to plan a harvesting schedule for the following days. A view of the coffee field, created from a composite of images, is shown in Figure 2. More details about the experiment can be found in References 3 and 4 .

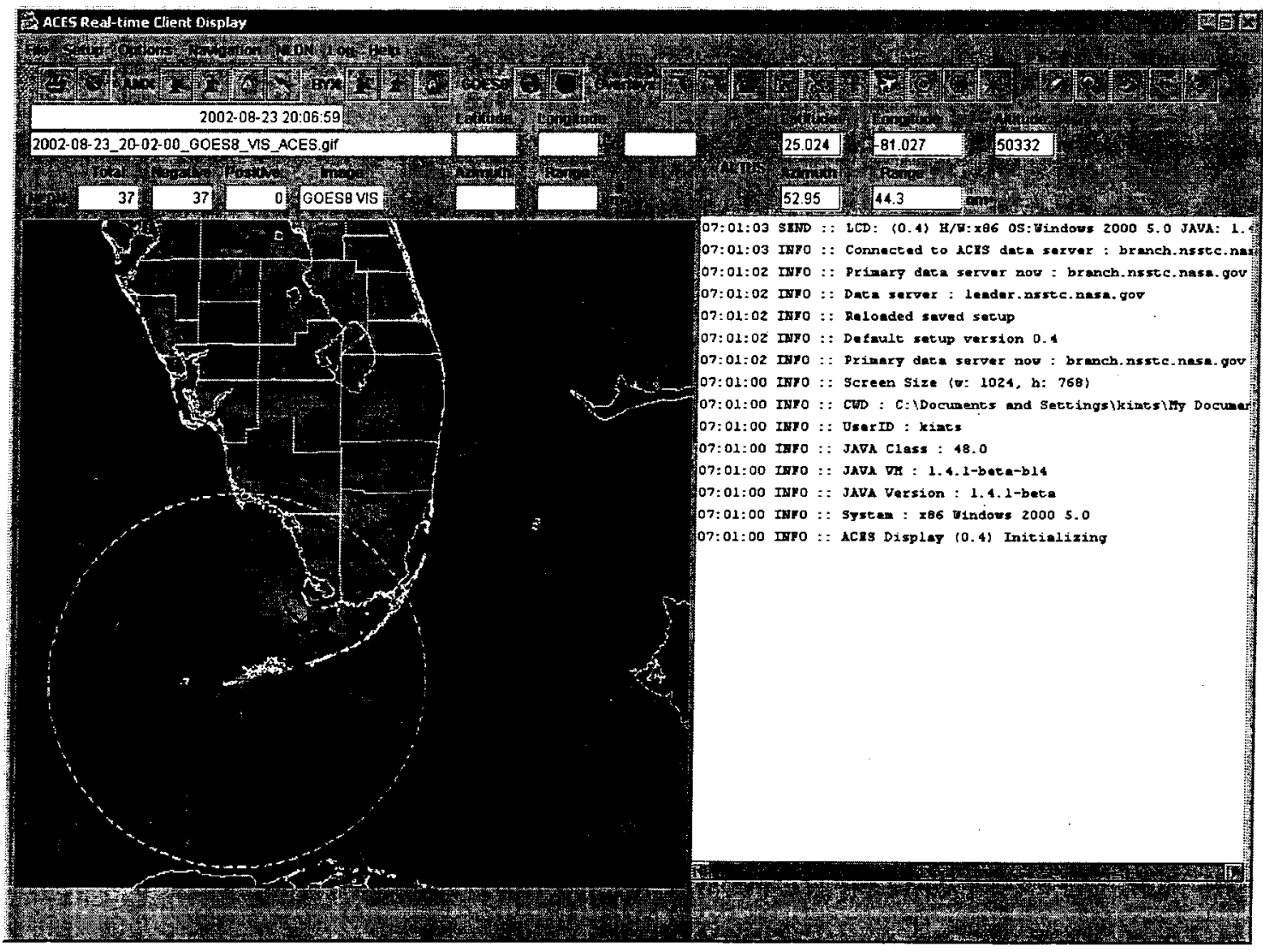

Figure 1: ACES Display Screen Showing Location of Altus ${ }^{\text {TM }}$ Relative to Weather 


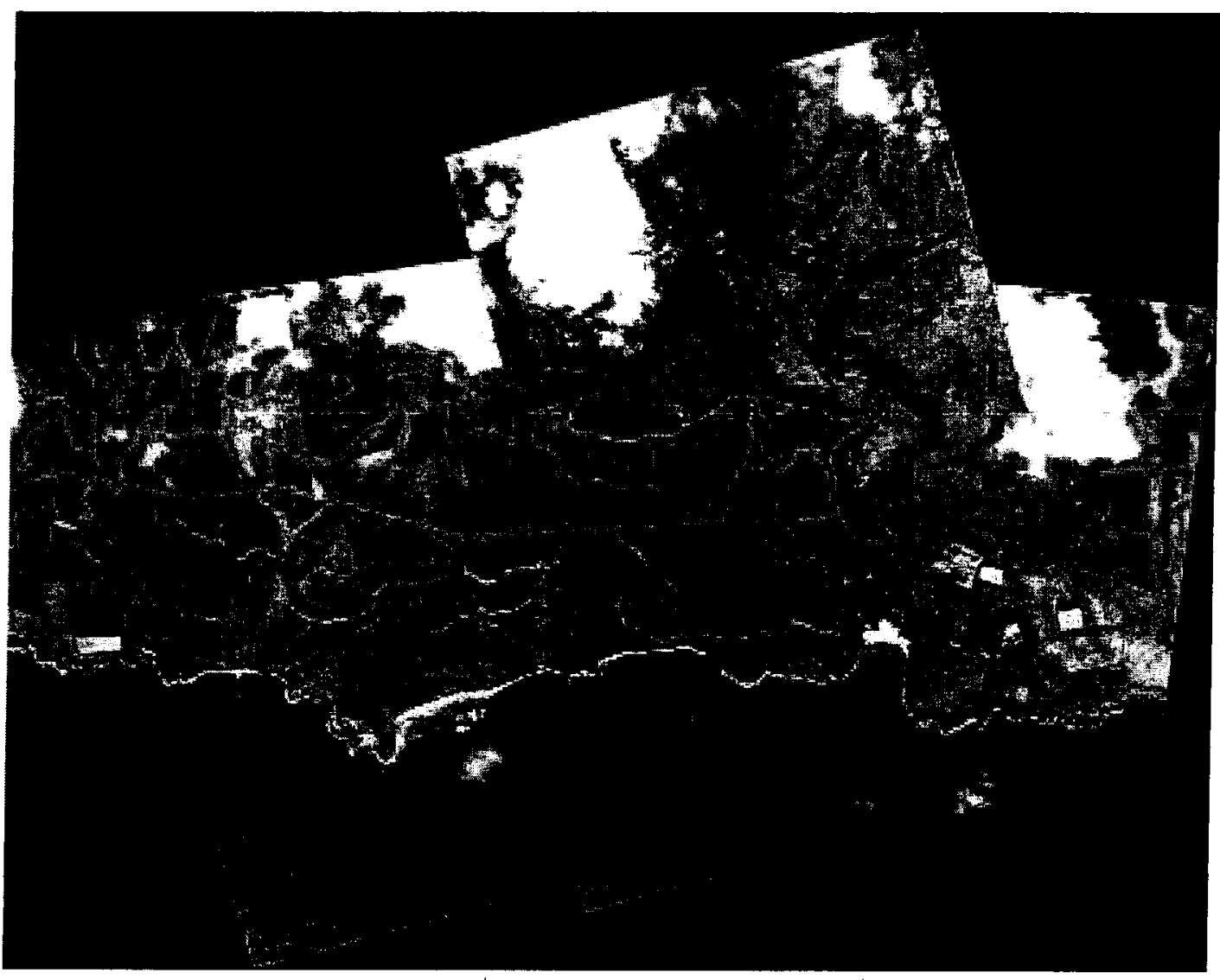

Figure 2: Kauai Coffee Plantation - Composite of High Resolution Visible Imagery

Payload design and operation

When flying on a UAV, the sensor payload must rely on either autonomous or remote control to operate when needed, as there is no one on board to control the instruments. In both the ACES and Coffee experiments, the sensors were operated remotely, i.e., with commands from the scientists on the ground. For ACES, the command signals were transmitted via the aircraft data telemetry system. For UAV Coffee, the command signals were transmitted directly to the instruments by wireless ethernet.

Because a scientist cannot be on-board the aircraft to check status, turn instruments on or off, or to collect data, these functions must all be designed in and tested in advance. This is a major activity for UAV science projects. In addition, if real-time data are needed or desired on the ground, then a suitable data transmission system is also needed.
Fortunately for both experiments, early versions of the sensor payloads had already been demonstrated to operate remotely on either manned platforms or UAVs prior to these missions. This made final design easier because of familiarity with the instruments.

The ACES science payload included a variety of electric, magnetic and optical sensors to obtain a detailed view of the electromagnetic processes within and around thunderstorms throughout their life cycles. In addition, a large variety of observational and forecast meteorological data products (i.e., observational products included Key West and Miami WSR-88D weather radar, GOES satellite images, and cloud-to-ground lightning from the National Lightning Detection Network) obtained through the Internet were utilized by the ACES project. These data were combined with the aircraft status data to aid in both

3

American Institute of Aeronautics and Astronautics 
mission planning and execution. This successful combination of data, along with the partnering of scientist and pilot together in the ground station is one of the highlights of both experiments.

Two camera system payloads were used for the UAV Coffee Mission - one a high-resolution visible system and the other a multispectral infrared camera. These payloads were selected to be complementary in spectral and spatial coverage of the plantation, and to provide a degree of redundancy in the primary data product. The payloads were configured for remote operation in the extreme pressure and temperature environment of the stratosphere, within the severe weight, volume, and power limitations of the aircraft. This downsizing of the payload was a major challenge for the overall project. Its successful operation was a major accomplishment. Testing on a manned platform was necessary for the cameras used in the Coffee mission, but there were timeconsuming problems to be solved related to the test aircraft and test sites that were not related to the UAV payload or conditions.
Integration and testing of the payload with the UAV must be done in advance of an actual mission, to test for performance of the instruments, command and control function, interference with the aircraft signals or operation, and data acquisition. A minimum of one such test flight is recommended. The ACES team flew a test flight out of El Mirage a month prior to the campaign. The Coffee team integrated instruments on the Pathfinder Plus on the ground in Southern California and test for interferences several months before the campaign, and then tested in Kauai during a "dress rehearsal" several days before the flight experiment.

\section{Platform Perfomance}

For the ACES experiment, MSFC chose the General Atomics Altus ${ }^{\text {TM }}$ UAV, which they had flown previously in an SBIR experiment with a similar payload. The Altus is powered by a dualturbocharged piston engine to achieve maximum altitude of $60,000 \mathrm{ft}$. It can fly above $50,000 \mathrm{ft}$ for up to 5 to 6 hours. It can carry a payload up to 150 kg. Altus is shown in Figure 3.

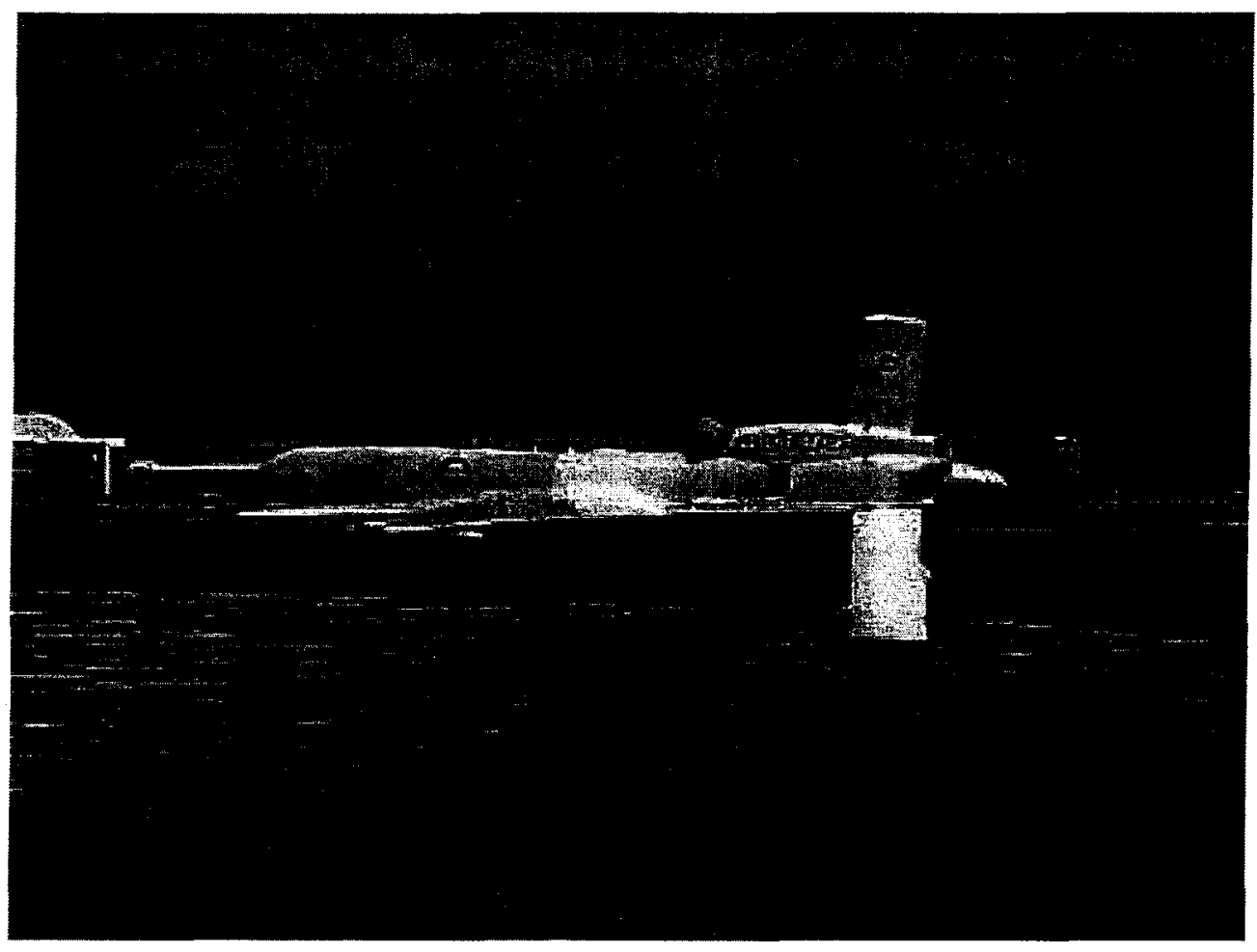

Figure 3: Altus on the Ramp at Naval Air Facility Key West 
Altus performed well in the experiment, in that it reached altitude and had sufficient endurance and range to reach thunderstorms of interest over south Florida from the mission base at Naval Air Facility Key West. However, the Altus engine is a one-of-a-kind system, which GA compares to maintaining a racecar engine. Maintenance requirements were high, and reliability relatively low, so that many hours or opportunities for science flights were lost to the science team while work was being done on the aircraft.

For the Coffee Optimization experiment, Clark University chose the solar-powered Pathfinder Plus. It had flown previously from the Pacific Missile Range Facility (PMRF) and, in fact, successfully flew several flights for another customer in the months immediately prior to the
Coffee mission. Pathfinder Plus can fly as high as $60,000 \mathrm{ft}$, but was only flown to $21,000 \mathrm{ft}$ for the Coffee mission, to give suitable resolution for the camera images of the colfee field. Pathfinder Plus is shown over Kauai in Figure 4.

On the day of the flight, Pathfinder Plus flew flawlessly and was carefully maneuvered to take advantage of openings in the clouds to get cloudfree images of the coffee plantation. All operations were successful. On the other hand, Pathfinder Plus, being a very light flying wing, is extremely limited in the conditions under which it can fly. Weather conditions at the PMRF forced cancellation of several hoped-for flight opportunities. Only one science flight (of the expected two or three) was completed.

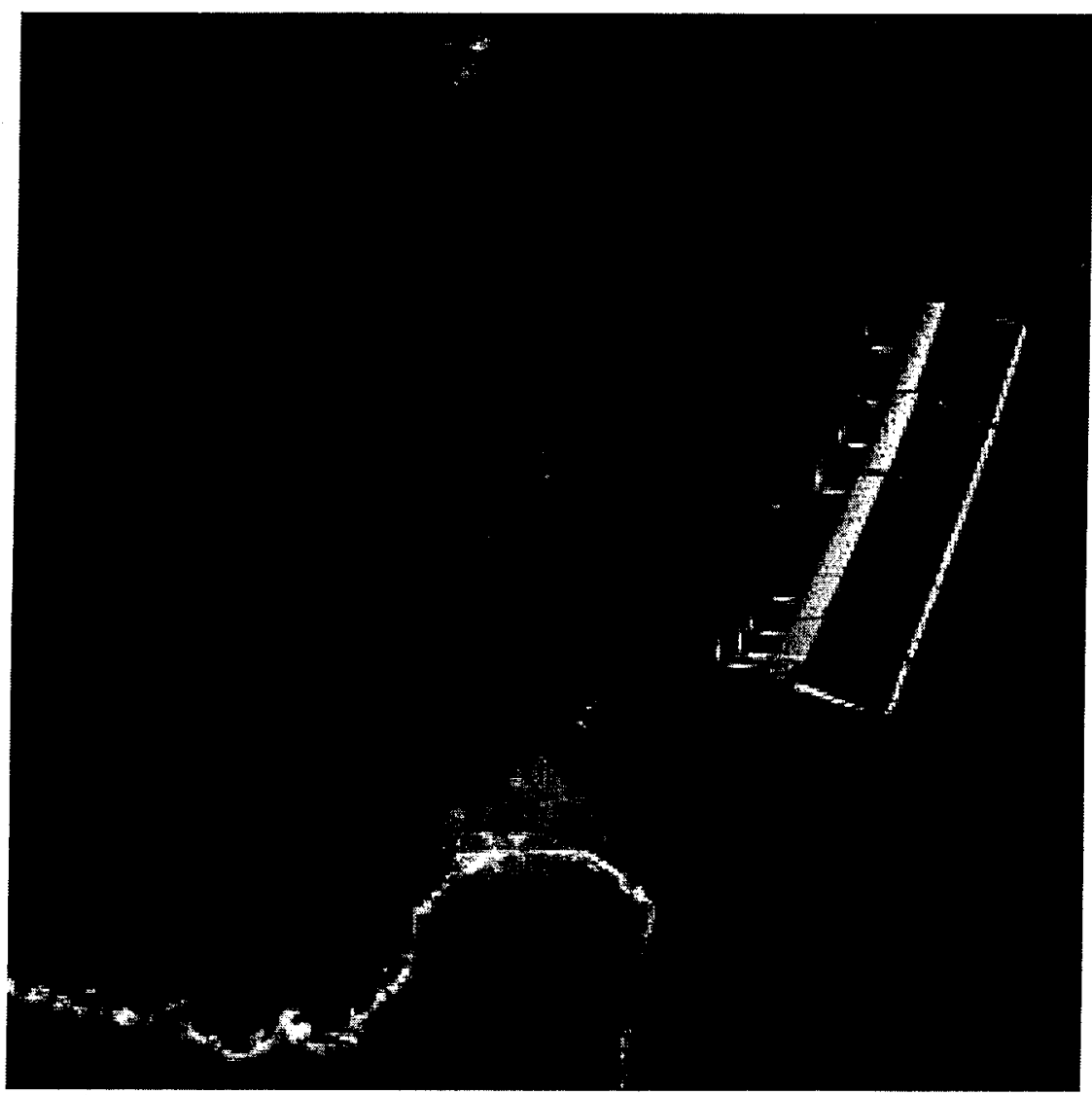

Figure 4: Pathfinder Plus over Kauai 
Some lessons learned relevant to UAV performance:

- UAVs with previous experience can be expected to perform as advertised in science missions.

- Flight conditions such as weather are a significant factor in the ability to fly some UAVs on any given day.

- An experienced flight team with adequate depth is desirable for success. This includes technicians, pilots, and a dedicated project manager.

- Thorough data on previous performance is needed for safety review success.

- Discuss with vendor previous lessons learned regarding risks and risk mitigation, to be adequately prepared in the fieid and avoid repeat problems.

- Integration with the payload and testing for interference should be performed as far in advance as possible.

- As these UAVs are not flown to the mission site, but are shipped, it is important to discuss shipping schedules and related issues in advance.

Flying in National Airspace

Both ACES and the Coffee mission flew from military facilities into restricted airspace and then transitioned to National Air Space. These facilities were chosen in part because they are government-owned and were believed to be easier to access by a NASA-sponsored program. Another reason was to facilitate the launch and landing in restricted airspace until the UAV could reach airspace controlled by Air Traffic Control (ATC).

Permission to fly in the National Air Space (not controlled by the military) is granted by the Federal Aviation Administration (FAA). The FAA is becoming more and more familiar with UAVs and in most regions is amenable to working with UAV teams to obtain a certificate of authorization (COA), which states where and under what conditions the UAV may enter the National Air Space. In both regions where experiments flew during UAVSDP, previous UAV experiments had been authorized. Previous flights of solar aircraft from PMRF had been authorized by Honolulu Center, and previous flights of the Aerosonde ${ }^{T M}$ UAV were authorized during Crystal-FACE in 2001 by Miami Center.

Working with the FAA was successful in both of these cases because the teams began discussions early to work out flight requirements. For ACES, although GA made the request for the COA, the science team was intimately involved in flight planning to ensure they could obtain the science data the required. For the Coffee mission, the science team employed a consultant (a former FAA employee) to manage the COA process. AeroVironment had always made this request themselves in the past, but the chosen approach was successful.

This program has shown that when things are working well, and good communications are maintained, that the FAA can be flexible. In fact the FAA allowed exceptions from approved flight plans in real time during both experiments, in order to enhance the science being pursued. During one of the later ACES flights, the FAA allowed Altus to transition to National Air Space before reaching $40,000 \mathrm{ft}$ (the formal restriction) so that the platform could more quickly reach a thunderstorm developing over the Everglades of Southwest Florida. Things had worked so well in previous flights, that the FAA was comfortable with this exception. Also, during the Coffee flight, because of the clouds over the Kauai Coffee plantation, and the desire to take pictures where there were no clouds, the FAA allowed Pathfinder Plus to follow a more-or-less random path over the plantation, in contrast to the filed flight plan. The actual flight path is shown in Figure 5. 


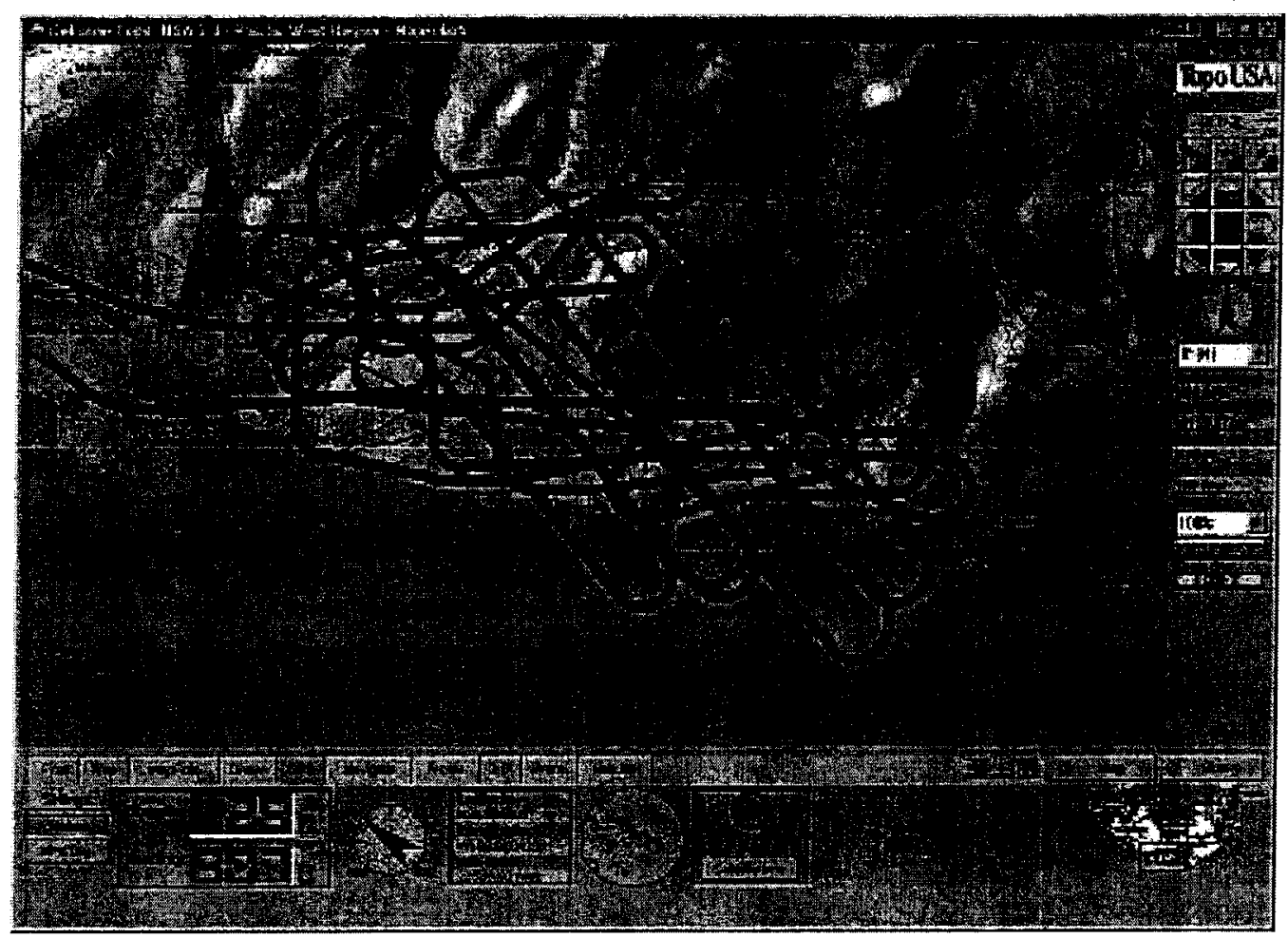

Figure 5: Pathfinder Plus Flight Path over Kauai Coffee Plantation

In determining a base for UAV flight operations, it is interesting to review the experiences of the UAVSDP teams. To date, the AeroVironment solar aircraft (Pathfinder, Pathfinder Plus, Centurion and Helios) have only flown from military bases (Edwards AFB and PMRF). The ACES team originally planned to fly from Patrick $A F B$, in part because of location, but also because it was deemed easier to draw up an MOU between NASA and the military than with a civil airport. Ultimately, ACES flew from Naval Air Facility Key West because there the team was able to obtain permission to fly with relative ease because the Navy was familiar with NASA aircraft campaigns and UAVs. The assumption that a military facility is a good base of operations should be challenged, however, as experience in this program showed offsetting disadvantages of flying from a military base. Some of the potential problems:

- Schedules are subject to override. Personnel change frequently. Safety concerns may be excessive. Radio frequencies are hard to obtain. Rules can change suddenly.

- Recognize that military facilities may not be able to provide fully auditable invoices.

- Plan to provide dedicated Internet networks for your team. Military firewalls are difficult to negotiate.

\section{Mission Management}

Mission planning is always an important part of a science mission. This is especially true for UAVs where the experience is limited. The science team needs to become intimately familiar with the UAV, its operation, and the service provider, in order to fully prepare for the mission. In the case of the UAVSDP program, both teams were required to prepare implementation plans prior to submitting final proposals. These implementation plans addressed most issues of mission planning, and hence a good deal of planning was done even before the projects began. The implementation plans could be considered a template for future mission planning activities. 
Some specific lessons learned:

- Establish roles and responsibilities early, ideally during the proposal phase.

- Establish liability in the proposal phase.

- Understand go/no-go criteria for both platform and science.

- If go/no-go criteria or other mission operations are based on previous missions, these should be reviewed for relevancy to the current mission.

Because of the nature of the NASA program, each team was headed by a Principal Investigator (PI), who was ultimately responsible for all aspects of mission management. There was no NASA "mission manager" assigned to these experiments to interface with the aircraft team. The PI was assisted by a project manager and other team members. The PI team interfaced directly with the UAV provider and other subcontractors. Although all team members in both experiments were committed to success, there were mission management challenges for both teams that arose from this approach.

Some strong suggestions for project management of future UAV missions are made here. One is that a full-time project manager on the science team is essential to handle all the mission arrangements. Similarly, a full-time project manager on the UAV team is also crucial, at least during pre-deployment and deployment periods. Having a committed partner at the flight facility is also important. Frequent communication is vital and team-building is essential. Systematic project management, (i.e., progress reporting of accomplishments, plans, risks and mitigation, schedules, and budgets) was required for these projects and was found to be a crucial factor in the success of both the science and UAV efforts.

\section{Concluding Advice}

Following are the most significant lessons learned, as articulated by the project teams:

- UAVs can provide unique platforms for science and applications.

- UAV systems are still immature and missions require careful attention and planning for success.

- Altus and its family of aircraft are ready for campaign-mode, whereas the solar aircraft, as currently configured, may be too weathersensitive for routine use.

- Real-time interaction of the PI with the pilot is easier and more productive on the ground.

- Real-time flight visualization tools are the future of suborbital science.

- Prepare a thorough implementation plan and follow it.

- Start early on all approvals.

- UAV payloads must be designed for remote or autonomous operation, and may need extensive testing. Plan schedule and resources accordingly. Use a payload with heritage.

- Communication within and outside the team is a challenge, and the KEY to success.

\section{The Future: Demonstration and Technology}

\section{Development}

NASA intends to continue the development and demonstration of UAVs, as they hold a key role in the future of Earth Science. Follow-on technology efforts are focused on achieving routine access to the National Air Space, and follow-on science missions will continue to show the unique value of UAVs in remote sensing, atmospheric science, disaster management and climate change research.

During the course of the UAVSDP program, some system and technology needs were identified. The systems needs include:

- A UAV capable of flying to $60,000 \mathrm{ft}$ with long endurance (at least $24 \mathrm{hrs}$ ) and adequate payload capacity (100's of pounds).

- Lower cost of UAV operations.

- More commercial UAV vendor options.

- Improved reliability of UAVs for high altitude, long endurance.

- Thorough documentation of aircraft systems (for safety review).

- Easier access to the National Air Space.

- More trained pilots.

In addition, specific UAV technology needs have been identified, including:

- Provide redundancy in all aircraft systems.

- Electrical hardening of the aircraft for meteorological work.

- On-going technology watch for advanced sensor systems. 
- Higher bandwidth data communications systems.

- O-T-H systems to move missions beyond the current line-of-sight limitation. These should have adequate data rate and coverage, and be affordable and compact.

- Develop technology to ease access to the NAS, such as see-and-avoid.

\section{Acknowledgements}

The authors acknowledge significant input and discussion regarding lessons learned with the Marshall Space Flight Center ACES team, the Clark University UAV Coffee team, General Atomics and AeroVironment personnel, and members of NASA's suborbital science group.

\section{$\underline{\text { References }}$}

1. Kim, Tony, "Altus Cumulus Electrification Study: A UAV Weather Science Demonstration," proceedings of Let's Fly, TAAC Conference 2002

2. "Spying on Storms," Aerospace America, January 2003

3. Herwitz, S.R., Johnson, L.F., Arvesen, J.C., Leung, J.G., Dunagan, S.E., 2002. Precision Agriculture as a Commercial Application for Solar-powered UAVs, proceedings $1^{\text {st }}$ AIAA UAV Conference, Portsmouth, VA.

4. Herwitz, S., L. Johnson, S. Dunagan, J. Brass, and $\mathrm{G}$. Witt, 2003. Orchestrating a near-realtime imaging mission in national airspace using a solar-powered UAV" this conference. 